\title{
Preoperative and Postoperative Hope Level of Patients with Newly Diagnosed Gastric Cancer
}

\author{
Wang Lingyan ${ }^{1, *}$, Shen Fang ${ }^{2}$, Wu Xiaodan ${ }^{2}$ \\ ${ }^{1}$ Department of Nursing Administration, Sun Yat-sen University Cancer Center, Guangzhou, China \\ ${ }^{2}$ Department of Gastrointestinal Surgery, Sun Yat-sen University Cancer Center, Guangzhou, China
}

Email address:

wangly@sysucc.org.cn (Wang Lingyan)

${ }^{*}$ Corresponding author

To cite this article:

Wang Lingyan, Shen Fang, Wu Xiaodan. Preoperative and Postoperative Hope Level of Patients with Newly Diagnosed Gastric Cancer. American Journal of Nursing Science. Vol. 8, No. 5, 2019, pp. 223-226. doi: 10.11648/j.ajns.20190805.13

Received: May 30, 2019; Accepted: July 25, 2019; Published: August 16, 2019

\begin{abstract}
Background: Hope is an essential factor to consider when caring for patients with cancer, It is a key component of coping with adversity and important for patient well-being. Treatment can relieve a patient's symptoms or cure the disease. It may also increase the patient's level of hope. Objective: This study aimed to explore the preoperative and postoperative hope level of patients with newly diagnosed gastric cancer, and identify how it changes over time. Methods: The study sample included 162 patients who diagnosed with gastric cancer in Sun Yat-sen University Cancer Center. The study was conducted between January 2018 to July 2018. Herth Hope Index was used to assess hope level of patients before surgery, one month and six months after surgery. Results: The score of hope level of the patients before surgery, one month and six months after surgery was $(34.20 \pm 3.75),(37.78 \pm 4.10)$ and $(39.16 \pm 4.73)$ respectively. It was found that the score was gradually increasing $(\mathrm{P}<0.01)$. In particular, the dimensions of temporality and future $(\mathrm{T})$ and interconnectedness (I) escalated obviously $(\mathrm{P}<0.01)$. Conclusions: This study indicates that the hope level of newly diagnosed gastric cancer patients was gradually improved after surgery. The finding remind us that we can take targeted measures to improve preoperative hope level of gastric cancer patients.
\end{abstract}

Keywords: Gastric Cancer, Hope Level, Longitudinal Study, Preoperative, Postoperative

\section{Introduction}

Gastric cancer is one of the most common malignancies in the world, and its morbidity and mortality rate rank among the top of all kinds of tumors. What's more, the incidence of gastric cancer tends to be younger in recent years [1-2] China is the high-epidemic area of gastric cancer worldwide [3]. Cancer not only causes physically problems, but also leads to psychological distress and poor adaptability [4-5].

Hope is a belief which convinces people that a positive, realistic, and expectable goal can be achieved in the future. Hope can be regarded as people's confidence in achieving their goals, as well as the cognition of the interdependence and interconnections between individuals' spirits [6]. Hope, long considered as an essential element for life, has been shown to be important among cancer patients in coping with illness and defusing death threats. It can alleviate pain and stress, and even affect the survival of the patients [7].

In the past, there have been many studies on the level of hope in cancer patients [6], but most of them are cross-sectional studies. They rarely reported on the characteristics of dynamic changes in the level of hope among gastric cancer patients at different cancer stages at home and abroad. Our study was designed to explore the dynamic changes of the hope level of newly diagnosed gastric cancer patients before and after surgical treatment, so as to provide theoretical basis for further formulating targeted interventions to improve the hope level of gastric cancer patients.

\section{Samples and Methods}

\subsection{Patients Enrollment}

Based on convenience sampling, we investigate patients who diagnosed with gastric cancer and intend to undergo surgery in Sun Yat-sen University Cancer Center from January 
2018 to July 2018. Patients were eligible for enrollment in the study if they: (1) had a histopathological diagnosis of gastric cancer; (2) were between 18 and 75 years of age; (3) knew that they had cancer; (4) had primary school education or above; (5) agreed to participate in this study. Exclusion criteria were: (1) mental illness in the past or at present (2) evidence of metastasis; (3) a diagnosis of other cancers; (4) serious systemic infections, severe anemia or other serious complications (such as heart, liver, kidney disease). Withdraw criteria were: (1) pelvic implantation and other distant metastases were found during operation; (2) not cooperate during the follow-up visits; (3) recurrence, metastasis or second tumor within 6 months after surgery.

In the study, 175 valid questionnaires of all initially issued questionnaires were retrieved. Totally, 162 patients completed the questionnaire for three times. Among 162 patients, 103 were male and 59 were female. The average age of all the patients were $52.2 \pm 12.0$. Thirty of 162 patients were primary school education, 60 were junior middle school education, 34 were high school or technical secondary school education, and 38 were college degree or above. One hundred and fifty-one of 162 patients were married, 11 were widowed or divorced. The family monthly income per capita of 26 patients was under one thousand yuan, 41 patients earned between one thousand yuan and two thousand yuan, 43 patients earned between two thousand yuan and three thousand yuan, 24 patients earned between three thousand yuan and four thousand yuan, 28 patients earned more than four thousand yuan. There were 70 employed, 54 unemployed and 38 retired patients. Forty-seven patients came from rural areas, 48 patients came from counties and 67 patients came from cities. There are 74 patients participated in medical insurance for employees or urban residents, 61 patients participated in rural cooperative medical insurance, 11 patients enjoyed free medical care and 16 patients were self-financed.

\subsection{Study Design}

\subsubsection{Demographic Questionnaire}

The self-designed questionnaire collected general information such as patients' gender, age, education, occupation, tumor site, cancer stage, surgical methods, chemotherapy regimens, etc.

\subsubsection{Herth Hope Index, $\mathrm{HHI}$ [8]}

Herth Hope Index was compiled by American scholar Herth in 1991 [9]. This study adopted the Chinese version of the
Herth Hope Index translated by Haiping Zhao [8] in 1999. The Herth Hope Index is composed of three subscales: temporality and future $(\mathrm{T})$, positive readiness and expectancy $(\mathrm{P})$ and interconnectedness (I). HHI contains 12 items, and each item is rated on a 4-point Likert scale that ranges from "strongly disagree (1)" to "strongly agree (4)." A total HHI score that can range from 12 to 48 points, and higher scores indicate higher level of hope. We use scores to identify the degree of hope (12 to 23 points indicate low hope; 24 to 35 points indicate moderately hopeful; and 36 to 48 points indicate high level of hope). The Cronbach's Alpha of internal consistency coefficient was 0.85 . Yanhua Wang [10] applied it to cancer patients with good reliability and validity.

\subsubsection{Survey Methods}

Questionnaire survey was used in this study. The uniform instructions were used in the survey to explain how to fill in the questionnaire and matters needing attention to the participants. The questionnaires were took back on the spot. Patients' clinical data of disease and treatment were obtained by referring to their medical records. For patients who were eligible for enrollment, they filled in the questionnaires two or three days before surgery for the first time. Then we approached participants for the second and third questionnaires 1 and 6 months after the surgery when they returned to the hospital for review or adjuvant chemotherapy.

\subsubsection{Statistical Analysis}

Statistical analysis was performed using SPSS13.0. Data for qualitative variables were reported as frequency, percentage (categorical data) and mean standard deviation (measurement data) for statistical description. Statistical analysis was performed using SPSS 20.0. Correlation analysis was used to explore the correlation between life quality and coping style of anxiety and depression.

\section{Results}

\subsection{Hope Level of Patients with Newly Diagnosed Gastric Cancer}

In this group, the preoperative hope level of patients with gastric cancer was mostly at the medium or low level, and it increased 1 and 6 months after surgery, mostly at the medium or high level (Table 1).

Table 1. Hope level of patients with newly diagnosed gastric cancer $(n=162)$.

\begin{tabular}{|c|c|c|c|c|c|c|}
\hline \multirow{2}{*}{ Groups } & \multicolumn{2}{|c|}{ Before surgery } & \multicolumn{2}{|c|}{ One month after surgery } & \multicolumn{2}{|c|}{ Six months after surgery } \\
\hline & Cases (n) & Percentage (\%) & Cases (n) & Percentage (\%) & Cases (n) & Percentage (\%) \\
\hline Low hope & 5 & 3.1 & 0 & 0 & 0 & 0 \\
\hline Moderately hopeful & 105 & 64.8 & 65 & 40.1 & 48 & 29.6 \\
\hline hopeful & 52 & 32.1 & 97 & 59.9 & 114 & 70.4 \\
\hline
\end{tabular}

\subsection{Dynamic Changes in the Level of Hope of Patients with Newly Diagnosed Gastric Cancer During Treatment}

In this group, the preoperative hope level of patients with gastric cancer was mostly at the medium level (34.20 \pm 3.75$)$, and it increased to high level after surgery. The dimensions of temporality and future (T) and interconnectedness (I) escalated rapidly. The dimension of positive readiness and expectancy (P) increased to a certain level and remained relatively stable (Table 2 ). 
Table 2. Dynamic changes in the level of hope of patients with newly diagnosed gastric cancer during treatment ( $n=162, \bar{x} \pm s)$.

\begin{tabular}{|c|c|c|c|c|c|}
\hline variable & Before surgery & One month after surgery & Six months after surgery & $\mathbf{F}$ & $\mathbf{P}$ \\
\hline Total score of hope level & $34.20 \pm 3.75$ & $37.78 \pm 4.10$ & $39.16 \pm 4.73$ & 59.749 & 0.000 \\
\hline temporality and future $(\mathrm{T})$ & $10.96 \pm 1.43$ & $12.14 \pm 1.54$ & $12.69 \pm 1.78$ & 49.909 & 0.000 \\
\hline positive readiness and expectancy $(\mathrm{P})$ & $12.25 \pm 1.41$ & $13.39 \pm 1.65$ & $13.73 \pm 1.81$ & 36.688 & 0.000 \\
\hline interconnectedness (I) & $11.00 \pm 1.41$ & $12.25 \pm 1.50$ & $12.75 \pm 1.70$ & 55.407 & 0.000 \\
\hline
\end{tabular}

\section{Discussion}

\subsection{Preoperative Hope Level of Newly Diagnosed Gastric Cancer Patients Needs to Be Improved}

Hope, considered as a positive expectation for achieving one's future goals (such as health), has been shown to have a positive impact on the individual's behavior and attitude. With the development of the application and research of positive psychology in our country, the society and medical community pay more and more attention to the study of hope. Hope, considered as an essential element during the treatment for every patients especially cancer patients, is the inner power that rules life. It encourages patients to overcome difficulties, alleviate the pain, and relieve the stress [9]. Our study shows that the preoperative hope level of patients with gastric cancer was mostly at the medium level (34.20 \pm 3.75$)$ and 30 of them were at low level which is consistent with some of the previous studies, but lower than the hope level (37.03 \pm 3.29$)$ reported by Caiping Zhao [11] which probably due to the backgrounds of the participants. In our study, patients are mostly from rural areas, in poor financial status and with lower education level. Rural patients with low education are backward in concept, and prone to fear of cancer. It is not easy for them to accept relevant knowledge about their disease, and their access to information about rehabilitation and health care is limited. Difficult to correctly understand the disease will lead to negative emotions. Patients in poor financial status endure great economic burden of medical care. They are afraid that they cannot afford the huge costs of surgery or follow-up treatment which results in lack of confidence in recovery and future life, and ultimately leads to a low level of hope. Some studies have also shown that economic income is positively correlated with the level of hope [12]. In addition, some scholars [13] believe that when people's hope is at stake, various measures will be adopted to protect and resurrect hope. However, patients with low education level and incomes in rural areas have few available resources and limited effective measures to resurrect hope. Therefore, their level of hope will be at a low level. Our results suggest that the hope level of newly diagnosed gastric cancer patients in the diagnostic stage needs to be improved. According to hope theory, patients with a high level of hope have more confidence in conquering cancer and comply better with treatment and rehabilitation, hence, patients' preoperative level of hope should be improved. Qualified case managers of cancer patients give them a comprehensive and systematic evaluation by case management, especially provide more counseling for patients who have low level of hope and limited resource so as to increase their confidence in treatment and rehabilitation, and improve prognosis. In addition, improvements in social support and cancer-related fatigue were also important for raising levels of hope [14-15].

\subsection{Analysis of Dynamic Changes in the Level of Hope of Newly Diagnosed Gastric Cancer Patients During Treatment}

Our study demonstrated that the preoperative hope level of patients with gastric cancer was mostly at the medium level $(34.20 \pm 3.75)$ and it increased to high level after surgery. The level of hope increased significantly at 1 month after surgery, and there was still a slight raise at 6 months after surgery. Surgery is a significant step in the treatment of gastric cancer. Patients who complete the surgery will gradually recover and return to normal life with remarkably improved hope level. But as time goes on, the effects of surgery and follow-up treatments on the body bring the patient symptom distress [16], so the hope level only rise slightly afterwards.

We also found that the scores of temporality and future (T), positive readiness and expectancy $(\mathrm{P})$, and interconnectedness (I) escalated rapidly at one month after surgery which were significantly higher than the preoperative level. The score of positive readiness and expectancy $(\mathrm{P})$ increased to a certain level and remained relatively stable one month after surgery. But the scores of temporality and future $(\mathrm{T})$ and interconnectedness (I) still increased significantly. The results indicated that most patients can take a positive attitude towards the reality and the future, and they can also re-establish relations with others and maintain intimate relationships after successful surgery. Due to early postoperative discomfort, the patients were troubled by some symptoms which limited the hope level rise to the maximum. When patients gradually recovered to health and alleviated the symptoms at 6 months after surgery, they would be more likely to maintain intimate relationships with others and more positive in attitude. Therefore, their scores of hope level continued to rise.

\section{Conclusions}

Our results suggest that patients' level of hope will change correspondingly with the development of disease and treatment. In the early stage of disease diagnosis, patients tend to lose confidence and hope due to the fear of the disease and side effects of treatments. As treatment progresses and the disease is properly treated, the patients readjusting the mindset and their hope level rise. As different dimension of hope level varies, we should take corresponding measures according to 
individual characteristics. For example, family members can be encouraged to provide more company and emotional support to patients, so as to increase their level of hope in interconnectedness.

\section{References}

[1] Zhang W Q, Zhang S W, et al. A report of cancer incidence and mortality from 38 cancer registries in China, 2007 [J]. China Cancer, 2011 (3), 162-169.

[2] Zhang W Q, Zhang S W, et al. Report of cancer incidence and mortality in China, 2009 [J]. China Cancer, 2013, 22 (01), 2-12.

[3] Chen W, Zheng R, et al. Cancer Statistics in China, 2015. CA Cancer J Clin, 2016, 66 (2), 115-132.

[4] Wu X D, Zhang M F, et al. Symptoms and influencing factors in patients undergoing chemotherapy after radical esophagectomy [J]. Chinese Journal of Nursing, 2013, 48 (11), 995-999.

[5] Zhang M F, Zheng M C, Liu W Y, et al. The influence of demographics, psychological factors and self-efficacy on symptom distress in colorectal cancer patients undergoing post-surgical adjuvant chemotherapy [J]. Eur J Oncol Nurs, 2015, 19 (1), 89-96.

[6] Vellone E, Rega M L, Galletti C, et al. Hope and related variables in Italian cancer patients [J]. Cancer Nurs, 2006, 29 (5), 356-366.

[7] Cutcliffe J. Critically ill patients' perspectives of hope [J]. Br J Nurs, 1996, 5 (11), 674, 687-690.
[8] Zhao H P, Wang J. Social support and hope of Hemodialysis Patients [J]. Chinese Journal of Nursing, 2000, 35 (5), 306-308.

[9] Herth K. Development and refinement of an instrument to measure hope [J]. Sch Inq Nurs Pract, 1991, 5 (1), 39-51, 53-56.

[10] Wang Y H. Study on feasibility of Chinese version of the Herth hope index for cancer patients [J]. Chinese Nursing Research, 2010, 24 (1), 20-21.

[11] Zhao C P, Fang J Q, et al. The relationship among hope level, personality, anxiety and depression in patients with gastric cancer [J]. Clin J Nurs, 2012, 47 (01), 65-67.

[12] Li Y M, Ma J W, Xue S O, et al. Investigation and analysis of the hope level of patients with esophageal cancer in a hospital [J]. General nursing, 2019, 17 (11), 1396-1397.

[13] $\mathrm{Wu} \mathrm{J} \mathrm{J.} \mathrm{The} \mathrm{relevance} \mathrm{between} \mathrm{quality} \mathrm{of} \mathrm{life,} \mathrm{level} \mathrm{of} \mathrm{hope} \mathrm{and}$ coping style of inpatients with colorectal cancer [D]. Henan University, 2011.

[14] Liang S, Chen Y Y, Xu X H, et al. A study on the correlation between quality of life and coping style and social support in patients with head and neck tumors [J]. Journal of nursing, 2017, 24 (10), 62-66.

[15] Xie Y M. Study on the relationship between hope level and cancer-related fatigue and negative emotions in breast cancer patients [J]. International journal of nursing, 2016, 35 (6), 817-819.

[16] Wu X D, Zhang M F, et al. Correlationships between symptom distress, anxiety and depression in esophageal cancer patients undergoing chemotherapy after radical esophagectomy [J]. J of Nurs Sci, 2013, 28 (6), 72-75. 